\title{
Association of lifestyle factors with blood lipids and inflammation in adults aged 40 years and above: a population-based cross- sectional study in Taiwan
}

\author{
Miriam Adoyo Muga ${ }^{1}$, Patrick Opiyo Owili ${ }^{2,3}$, Chien-Yeh Hsu ${ }^{4,5}$ and Jane C.-J. Chao ${ }^{5,6,7^{*}}$ (1)
}

\begin{abstract}
Background: Lifestyle factors were associated with an increased risk of cardiovascular disease (CVD) occurrence. We explored the associations between lifestyle factors and CVD risk factors, and assessed the interactive effects of lifestyle factors on CVD risk factors.

Methods: A cross-sectional data of 114,082 (57,680 men and 56,402 women) middle-aged adults and elderly in Taiwan were collected from 2001 to 2010. Logistic regression analysis was used to assess the associations between lifestyle factors and CVD risk factors. The relative excess risk due to interaction (RERI) and the attributable proportion due to interaction were used to explore the interactive effect of lifestyle factors on CVD risk factors.

Results: The interaction between alcohol consumption and smoking exhibited an excess risk of high triglycerides $(\mathrm{RERI}=0.21 ; 95 \% \mathrm{Cl}: 0.14-0.29)$, and that of alcohol consumption and physical activity had an excess risk of high LDL-cholesterol (RERI $=0.11 ; 95 \% \mathrm{Cl}: 0.06-0.16)$ and high blood glucose (RERI $=0.05 ; 95 \% \mathrm{Cl}: 0.01-0.11)$. Alcohol consumption and vegetable-rich diet (intake of high vegetables with no or low meat) had an excess risk of high LDL-cholesterol and low HDL-cholesterol, but a reduced risk of high triglycerides (RERI $=-0.10 ; 95 \% \mathrm{Cl}$ : $-0.17-$ -0.04). Smoking and physical activity had an increased risk of high blood glucose and a reduced risk of low HDLcholesterol. Smoking and vegetable-rich diet reduced the risk of high triglycerides (RERI $=-0.11 ; 95 \% \mathrm{Cl}$ : $-0.18-$ $-0.04)$, high blood glucose (RERI $=-0.14 ; 95 \% \mathrm{Cl}$ : $0.21--0.07)$ and low HDL-cholesterol (RERI $=-0.10 ; 95 \%$ $\mathrm{Cl}:-0.19--0.01)$.

Conclusions: The interaction between smoking, alcohol consumption, physical activity and diet were associated with lipid profile and blood glucose, hence there was an interaction between these lifestyle factors in an additive scale. Public health promotion should therefore consider multifaceted promotional activities that are likely to make a positive impact on the health status of the Taiwanese population.
\end{abstract}

Keywords: Lifestyle factors, Blood lipids, Inflammation, Cardiovascular disease

\footnotetext{
* Correspondence: chenjui@tmu.edu.tw

${ }^{5}$ Master Program in Global Health and Development, College of Public

Health, Taipei Medical University, Taipei, Taiwan

${ }^{6}$ School of Nutrition and Health Sciences, College of Nutrition, Taipei Medical

University, 250 Wu-Hsing Street, Taipei 110, Taiwan

Full list of author information is available at the end of the article
}

(c) The Author(s). 2019 Open Access This article is distributed under the terms of the Creative Commons Attribution 4.0 International License (http://creativecommons.org/licenses/by/4.0/) which permits unrestricted use, distribution, and reproduction in any medium, provided you give appropriate credit to the original author(s) and the source, provide a link to the Creative Commons license, and indicate if changes were made. The Creative Commons Public Domain Dedication waiver (http://creativecommons.org/publicdomain/zero/1.0/) applies to the data made available in this article, unless otherwise stated. 


\section{Background}

In recent years, heart disease has become the second leading cause of death after malignant neoplasms in Taiwan [1]. Moreover, the prevalence of overweight and obesity among adults in Taiwan increased obviously from $33.2 \%$ during $1993-1996$ to $43.0 \%$ during $2013-$ 2014 [2]. Unhealthy lifestyle habits such as smoking, alcohol consumption, physical inactivity and poor dietary habits have been considered to be associated with an increased risk of overweight and cardiovascular disease (CVD) [3, 4].

Low level of high-density lipoprotein-cholesterol (HDL-C) and raised levels of triglycerides (TG), total cholesterol (TC), low-density lipoprotein-cholesterol (LDL-C), C-reactive protein (CRP) and high blood glucose have been found to be the risk factors of CVD [58]. In addition, hypertension and obesity have been consistently associated with the risk factors of CVD [7, 9]. However, good lifestyle habits such as regular exercise and healthy dietary habits have been known as protective factors against hyperlipidemia as well as CVD occurrence and mortality [10-14], while cigarette smoking is known to have a negative effect on blood lipids [15-17]. The association between lifestyle factors and etiology of lipid abnormalities has not been fully understood, partly because of the limitations of study design and/or analytical strategies used. Moreover, the interactive effects of these lifestyle factors on blood lipids and inflammation are not clear in literature.

Therefore, our study assessed the associations between lifestyle factors which include smoking, alcohol consumption, physical activity and dietary habit and CVD biochemical risk factors which include TG, TC, LDL-C, HDL-C, CRP and blood glucose among middle-aged adults and elderly in Taiwan. Further, we explored the interactive effects of lifestyle factors on CVD risk factors in the middle-aged and elderly population.

\section{Methods}

\section{Study design and population}

We used a cross-sectional data collected by Mei Jau (MJ) Health Management Institute and screening centers in Taiwan between 2001 and 2010. The MJ group is a private health screening organization offering comprehensive health screening services and healthcare management consultations. The participants used in this study were individuals who visited the facilities between 2001 and 2010. The participants had answered a structured questionnaire prior to physical examination and signed an informed consent to use their data for research purposes without disclosure of personal information. A total of 765,064 adults aged $\geq 40$ years visited MJ health screening centers in Taipei, Taoyuan, Taichung or Kaohsiung in a 10-year period. If the participants visited $\mathrm{MJ}$ health screening centers more than once with multiple entries $(n=404,829)$, we only used the data at their first visit because they had repeated measurements of biomarkers which were excluded. We also excluded those who had some diseases (cancer, diabetes, renal or liver diseases) $(n=77,160)$, certain drug use (psychiatric or hypolipidemic drugs $)(n=43,713)$ or missing data $(n=125,280)$. Therefore, a total of 114,082 (57,680 men, $50.6 \%$; 56,402 women, 49.4\%) middle-aged adults and elderly aged $\geq 40$ years participated in the study. The participants with these diseases and those who were taking lipid lowering drugs were excluded because the medication would confound the findings in this study. The exclusion criteria minimized the biases associated with adjustment of lifestyle as a result of being aware of their health status. The demographic and lifestyle data of the participants were collected using standardized and validated questionnaires, and biochemical data were analyzed from collected blood specimens of the participants.

\section{Ethical consideration}

An informed consent was signed by the participants before health screening to agree that anonymized data would be used only for academic purpose. This study was approved by the Taipei Medical University-Joint Institutional Review Board.

\section{Outcomes: biochemical measurements}

Before drawing blood from the participants, overnight fasting (12-14h) was obligatory. Blood specimens were analyzed at the central laboratory of MJ. Reagents from Randox Laboratories Limited were used to measure the levels of fasting blood glucose and blood lipids (TG, TC and HDL-C). Blood LDL-C levels were determined by using the Friedewald formula (LDL-C $=$ TC - HDL-C TG/5) [18]. The reagent from Fortress Diagnostics was used to measure CRP levels. Repeated blood tests were performed to ensure accuracy. We used clinically defined levels to identify the participants at a potential high risk of CVD as follows: TG $\geq 150 \mathrm{mg} / \mathrm{dL}(1.695 \mathrm{mmol} / \mathrm{L})$, $\mathrm{TC} \geq 200 \mathrm{mg} / \mathrm{dL} \quad(5.18 \mathrm{mmol} / \mathrm{L}), \quad \mathrm{LDL}-\mathrm{C} \geq 100 \mathrm{mg} / \mathrm{dL}$ (2.59 mmol/L), HDL-C $\leq 40 \mathrm{mg} / \mathrm{dL} \quad(1.036 \mathrm{mmol} / \mathrm{L})$, $\mathrm{CRP} \geq 1.0 \mathrm{mg} / \mathrm{L}(9.52 \mathrm{nmol} / \mathrm{L})$ or fasting blood glucose $\geq 100 \mathrm{mg} / \mathrm{dL}(5.556 \mathrm{mmol} / \mathrm{L})[19,20]$.

\section{Predictors: lifestyle factors}

Information of lifestyle factors including smoking, alcohol consumption, physical activity and dietary habits were collected using the questionnaire when the participants visited the screening center before health screening. Smoking was categorized as non-smoker, often inhale secondhand smoke, quit smoking in less than 1 year and smoker. Alcohol consumption was divided as not drinking, $\leq 1$ glass/week, 2-3 glasses/week and $\geq 4$ 
glasses/week. Physical activity including any form of physical exercise was categorized as none, $\leq 2 \mathrm{~h} /$ week, $3-6 \mathrm{~h} /$ week and $\geq 7 \mathrm{~h} /$ week. Dietary habits were categorized into 3 groups: (1) intake of no or low vegetables with high meat, (2) intake of moderate vegetables with moderate meat and (3) intake of high vegetables with no or low meat. The dietary servings and frequency (i.e., per week or per day) was assessed using the food frequency questionnaire (FFQ) with 22 food items [21]. For example, intake of vegetables and root crops was assessed using the number of bowls per day (i.e., a bowl equals $11 \mathrm{~cm}$ in diameter). On the other hand, intake of fruits was assessed using servings per day. The other food items including meat and organ meats were assessed using servings per week. Vegetables or meat dietary pattern was determined by principal component analysis from the 22 food groups without overlapping. The food scores of each dietary pattern were summed up from 1 to 5 defined as the lowest to highest intake frequency of each food group, and intake of each dietary pattern was then divided into tertiles of different consumption indicating no/low intake, moderate intake or high intake.

\section{Potential confounders: other factors}

Other factors included in our analyses were gender, age groups $(40-44,45-49,50-54,55-59,60-64$ and $\geq 65$ years), education (primary school, high school and college) and marital status (single, married and widowed or divorced). The health characteristics included history of CVD (no or yes), systolic blood pressure (normal or elevated systolic blood pressure $\geq 120 \mathrm{mmHg}$ ) and diastolic blood pressure (normal or elevated diastolic blood pressure $\geq 80 \mathrm{mmHg}$ ) [22]. Weight status was defined as body mass index $(\mathrm{BMI})<24 \mathrm{~kg} / \mathrm{m}^{2}$ for non-overweight/ obese or BMI $\geq 24 \mathrm{~kg} / \mathrm{m}^{2}$ for overweight/obese using national criteria developed by the Ministry of Health and Welfare in Taiwan [23], and waist circumference was measured in centimeter. All these sociodemographic factors and health characteristics were considered potential confounders.

\section{Statistical analysis}

The statistical analyses included descriptive, analytical and interactive analyses. The characteristics of lifestyle factors were compared for different CVD risk factors by using chi-square test for categorical data and linear regression analysis for continuous data. Logistic regression analysis was used to assess the association between lifestyle factors and CVD risk factors. Two models were analyzed to produce the odds ratios (ORs) with 95\% confidence interval $(\mathrm{CI})$; model 1 was unadjusted and model 2 was adjusted for all the confounders in our study (i.e., sociodemographic and health factors).
The interactive effect of lifestyle factors on CVD risk factors in the additive scale was determined by relative excess risk due to interaction (RERI) and attributable proportion due to interaction (AP) to estimate the risk ratio from the adjusted ORs. Information on RERI and AP has been published elsewhere [24-26]. Each lifestyle factor was dichotomized: smoking vs not smoking, drinking alcohol vs not drinking, physical activity vs no physical activity and high vegetable consumption vs no or low vegetable consumption. The RERI estimates the extra risk due to interaction (RERI $\approx \mathrm{OR}_{11}-\mathrm{OR}_{10}-$ $\mathrm{OR}_{01}+1$ ) [27], when - RERI $<0$ suggests less than additivity or a negative interaction between the dependent and independent variables. For instant, if one independent variable combines alcohol consumption or smoking status, there could be an extra risk or a negative effect on the dependent variable. The proportion of a combined effect due to interaction was estimated by using $\mathrm{AP}\left(\mathrm{AP}=\mathrm{RERI} / \mathrm{OR}_{11}\right)$, when $-\mathrm{AP}<0$ also suggests less than additivity. Logistic regression matrix used with the 'ici' command in the Stata was to estimate the interaction effect and the 95\% CI. The variance and covariance of the coefficients were used to estimate the $95 \%$ CI because of our large sample size [28, 29]. All the analyses were performed by the Stata version 13.1 (StataCorp LLC, College Station, Texas) [30]. The $p \leq 0.05$ level was set for statistical significance.

\section{Results}

\section{Participants' characteristics}

Table 1 presents the characteristics of the participants by lifestyle factors. All the characteristics were significantly different $(p<0.05)$ between dichotomized lifestyle factors, except for the history of CVD between dichotomized smoking status $(p=0.262)$ and drinking alcohol status $(p=0.640)$, CRP levels between dichotomized physical activity $(p=0.430)$ and diastolic blood pressure between dichotomized vegetable diet $(p=0.288)$.

\section{Associations between lifestyle factors and CVD risk factors}

The unadjusted and adjusted ORs of CVD biomarkers by lifestyle factors are shown in Table 2. Significant associations between lifestyle factors and most of CVD risk factors were observed in both unadjusted and adjusted models. The adjusted model revealed that smoking significantly increased the development of high TG $(\mathrm{OR}=$ 1.44; 95\% CI: $1.38-1.50 ; p \leq 0.001)$, TC (OR $=1.08$; 95\% CI: $1.04-1.12 ; p \leq 0.001)$, CRP (OR $=1.33$; 95\% CI: 1.19 $1.47 ; p \leq 0.001)$, blood glucose (OR $=1.49 ; 95 \% \mathrm{CI}: 1.43-$ $1.55 ; p \leq 0.001)$ and low HDL-C levels $(\mathrm{OR}=1.60$; $95 \%$ CI: $1.52-1.68 ; p \leq 0.001)$ compared with non-smoking. Additionally, those who quitted smoking attenuated all the CVD risk factors, except for blood TG level $(\mathrm{OR}=$ 
Table 1 Characteristics of participants by lifestyle factors

\begin{tabular}{|c|c|c|c|c|c|c|c|c|}
\hline & Lifestyle facto & $n=114,08$ & & & & & & \\
\hline & Smoking & & Drinking alcor & & Physical activi & & High vegetab & diet \\
\hline & $n(\%)$ & $P$-value & $n(\%)$ & $P$-value & $n(\%)$ & $P$-value & $n(\%)$ & $P$-value \\
\hline All & $33,108(29.0)$ & & $30,264(26.5)$ & & $52,305(45.9)$ & & $62,089(54.4)$ & \\
\hline Triglycerides & & $<0.001$ & & $<0.001$ & & $<0.001$ & & $<0.001$ \\
\hline Normal & $22,446(25.9)$ & & $21,174(24.4)$ & & $39,375(45.4)$ & & $48,361(55.7)$ & \\
\hline High ( $\geq 1.695$ mmol/L) & $10,662(39.0)$ & & $9090(33.3)$ & & $12,930(47.4)$ & & $13,728(50.3)$ & \\
\hline Total cholesterol & & $<0.001$ & & 0.013 & & $<0.001$ & & $<0.001$ \\
\hline Normal & $16,921(28.6)$ & & $15,514(26.2)$ & & $27,569(46.6)$ & & $32,838(55.5)$ & \\
\hline High ( $\geq 5.18 \mathrm{mmol} / \mathrm{L})$ & $16,187(29.5)$ & & $14,750(26.9)$ & & $24,736(45.1)$ & & $29,251(53.3)$ & \\
\hline LDL-C & & $<0.001$ & & 0.003 & & 0.016 & & $<0.001$ \\
\hline Normal & $8178(26.6)$ & & $7965(25.9)$ & & $13,923(45.3)$ & & $17,571(57.1)$ & \\
\hline High ( $\geq 2.59 \mathrm{mmol} / \mathrm{L})$ & $24,930(29.9)$ & & $22,299(26.8)$ & & $38,382(46.1)$ & & $44,518(53.4)$ & \\
\hline $\mathrm{HDL}-\mathrm{C}$ & & $<0.001$ & & $<0.001$ & & $<0.001$ & & $<0.001$ \\
\hline Normal & $26,767(26.7)$ & & $25,406(25.3)$ & & $44,382(44.2)$ & & $55,507(55.3)$ & \\
\hline High ( $\geq 1.036 \mathrm{mmol} / \mathrm{L})$ & $6341(46.3)$ & & $4858(35.5)$ & & $7923(57.9)$ & & $6582(48.1)$ & \\
\hline CRP & & $<0.001$ & & $<0.001$ & & 0.430 & & 0.014 \\
\hline Normal & $32,012(28.9)$ & & $29,321(26.4)$ & & $50,851(45.8)$ & & $60,456(54.5)$ & \\
\hline High ( $\geq 9.52 \mathrm{nmol} / \mathrm{L})$ & $1096(35.1)$ & & $943(30.2)$ & & $1454(46.5)$ & & $1633(52.3)$ & \\
\hline Fasting blood glucose & & $<0.001$ & & $<0.001$ & & $<0.001$ & & $<0.001$ \\
\hline Normal & $15,478(22.8)$ & & $15,842(23.3)$ & & $29,631(43.6)$ & & $38,955(57.3)$ & \\
\hline High ( $\geq 5.556$ mmol/L) & $17,630(38.3)$ & & $14,422(31.3)$ & & $22,674(49.3)$ & & $23,134(50.3)$ & \\
\hline Gender & & $<0.001$ & & $<0.001$ & & $<0.001$ & & $<0.001$ \\
\hline Female & $5449(9.7)$ & & $6419(11.4)$ & & $24,832(44.0)$ & & $32,767(58.1)$ & \\
\hline Male & $27,659(48.0)$ & & $23,845(41.3)$ & & $27,471(47.9)$ & & $29,322(50.8)$ & \\
\hline Age group (years) & & $<0.001$ & & $<0.001$ & & $<0.001$ & & $<0.001$ \\
\hline $40-44$ & $10,111(31.4)$ & & $8060(25.0)$ & & $13,934(43.3)$ & & $14,875(46.2)$ & \\
\hline $45-49$ & $7296(29.9)$ & & $6652(27.3)$ & & $10,930(44.8)$ & & $12,881(52.8)$ & \\
\hline $50-54$ & $5151(28.2)$ & & $5065(27.7)$ & & $8363(45.8)$ & & 10,331 (56.6) & \\
\hline $55-59$ & $3709(26.3)$ & & $3788(26.8)$ & & $6168(43.7)$ & & 8458 (59.9) & \\
\hline $60-64$ & $2934(26.4)$ & & $3017(27.1)$ & & $5741(51.6)$ & & $6708(60.3)$ & \\
\hline$\geq 65$ & $3907(28.0)$ & & $3682(26.4)$ & & $7169(51.3)$ & & $8836(63.3)$ & \\
\hline Education & & $<0.001$ & & $<0.001$ & & $<0.001$ & & $<0.001$ \\
\hline Primary school & $6722(26.3)$ & & $6450(25.3)$ & & $13,284(52.1)$ & & $13,875(54.4)$ & \\
\hline High school & $11,785(33.0)$ & & $11,167(31.3)$ & & $16,734(46.9)$ & & $18,458(51.7)$ & \\
\hline College & $14,601(27.6)$ & & $12,647(23.9)$ & & $22,287(42.3)$ & & $29,756(56.3)$ & \\
\hline Marital status & & $<0.001$ & & $<0.001$ & & $<0.001$ & & $<0.001$ \\
\hline Single & $950(21.9)$ & & $761(17.5)$ & & $1417(32.7)$ & & $2283(52.6)$ & \\
\hline Married & $29,337(30.1)$ & & $26,777(27.5)$ & & $45,187(46.4)$ & & $52,807(54.3)$ & \\
\hline Widow/Divorced & $2821(22.7)$ & & $2726(22.0)$ & & $5701(46.0)$ & & $6999(56.4)$ & \\
\hline History of CVD & & 0.262 & & 0.640 & & $<0.001$ & & $<0.001$ \\
\hline No & $31,814(29.1)$ & & $29,064(26.5)$ & & $49,956(45.6)$ & & $59,459(54.3)$ & \\
\hline Yes & $1294(28.3)$ & & $1200(26.2)$ & & $2349(51.3)$ & & $2630(54.4)$ & \\
\hline Weight status & & $<0.001$ & & $<0.001$ & & 0.003 & & $<0.001$ \\
\hline Non-overweight/obese & $20,656(26.4)$ & & $18,947(24.3)$ & & $35,591(45.6)$ & & $44,286(56.7)$ & \\
\hline
\end{tabular}


Table 1 Characteristics of participants by lifestyle factors (Continued)

\begin{tabular}{|c|c|c|c|c|c|c|c|c|}
\hline & \multicolumn{8}{|c|}{ Lifestyle factors, $n=114,082$} \\
\hline & \multicolumn{2}{|l|}{ Smoking } & \multicolumn{2}{|c|}{ Drinking alcohol } & \multicolumn{2}{|c|}{ Physical activity } & \multicolumn{2}{|c|}{ High vegetable diet } \\
\hline & $n(\%)$ & $P$-value & $n(\%)$ & $P$-value & $n(\%)$ & $P$-value & $\overline{n(\%)}$ & $P$-value \\
\hline Overweight/obese & $12,452(34.6)$ & & $11,317(31.5)$ & & $16,714(46.5)$ & & $17,803(49.5)$ & \\
\hline Waist circumference $(\mathrm{cm}), \mathrm{M}(\mathrm{SD})^{\mathrm{a}}$ & $83.0(9.2)$ & $<0.001$ & $82.7(9.2)$ & $<0.001$ & $79.4(9.5)$ & $<0.001$ & $78.3(9.4)$ & $<0.001$ \\
\hline Systolic blood pressure & & 0.005 & & $<0.001$ & & $<0.001$ & & $<0.001$ \\
\hline Normal & $16,081(28.6)$ & & $13,628(24.3)$ & & $24,359(43.4)$ & & $30,176(53.7)$ & \\
\hline$\geq 120 \mathrm{mmHg}$ & $17,027(29.4)$ & & $16,636(28.7)$ & & $27,946(48.2)$ & & $31,913(55.1)$ & \\
\hline Diastolic blood pressure & & $<0.001$ & & $<0.001$ & & $<0.001$ & & 0.288 \\
\hline Normal & $22,378(27.8)$ & & $19,495(24.3)$ & & $36,204(45.0)$ & & $43,843(54.5)$ & \\
\hline$\geq 80 \mathrm{mmHg}$ & $10,730(31.9)$ & & $10,769(32.0)$ & & $16,101(47.8)$ & & $18,246(54.2)$ & \\
\hline
\end{tabular}

${ }^{a}$ Linear regression analysis was used to determine the mean (M) difference and standard deviation (SD)

LDL-C, low-density lipoprotein-cholesterol; HDL-C, high-density lipoprotein-cholesterol; CRP, C-reactive protein; CVD, cardiovascular disease

The $p$-values $\leq 0.05$ are presented in bold

1.06; 95\% CI: 1.01-1.12; $p \leq 0.05)$. Drinking alcohol $(\geq 4$ glasses/week) was more likely to have high TG (OR = 1.25; 95\% CI: $1.18-1.33 ; p \leq 0.001)$, TC $(\mathrm{OR}=1.14 ; 95 \%$ CI: $1.08-1.20 ; p \leq 0.001)$ and CRP levels (OR $=1.22 ; 95 \%$ CI: $1.05-1.42 ; p \leq 0.01$ ), but less likely to have high LDL$\mathrm{C}(\mathrm{OR}=0.79 ; 95 \% \mathrm{CI}: 0.74-0.85 ; p \leq 0.001)$, blood glucose $(\mathrm{OR}=0.68 ; 95 \% \mathrm{CI}: 0.64-0.72 ; p \leq 0.001)$ and low HDL-C levels (OR $=0.64$; 95\% CI: 0.59-0.69; $p \leq 0.001)$ than non-drinking. Those who were engaged in physical activity for more than $7 \mathrm{~h}$ per week were less likely to have high TG $(\mathrm{OR}=0.86 ; 95 \% \mathrm{CI}: 0.81-0.91 ; p \leq 0.001)$, TC $(\mathrm{OR}=0.88 ; 95 \% \mathrm{CI}: 0.84-0.93 ; p \leq 0.001)$ and CRP levels $(\mathrm{OR}=0.79 ; 95 \% \mathrm{CI}$ : $0.68-0.92 ; p \leq 0.01)$, but were more likely to have low HDL-C level $(\mathrm{OR}=1.45$; $95 \% \mathrm{CI}$ : $1.35-1.57 ; p \leq 0.001)$ and high blood glucose level (OR = 1.07; 95\% CI: $1.01-1.13 ; p \leq 0.05)$ than those who were not. Those who had a diet high in vegetables and no/low meat were less likely to have high TG $(\mathrm{OR}=0.94 ; 95 \%$ CI: $0.91-0.97 ; p \leq 0.001)$, TC (OR $=0.85$; 95\% CI: $0.83-$ $0.88 ; p \leq 0.001)$, LDL-C $(\mathrm{OR}=0.81 ; 95 \% \mathrm{CI}: 0.78-0.84$; $p \leq 0.001)$ and blood glucose levels (OR $=0.84 ; 95 \% \mathrm{CI}$ : $0.81-0.87 ; p \leq 0.001)$, and less likely to have low HDL-C level (OR $=0.92$; 95\% CI: $0.88-0.97 ; p \leq 0.001)$ than those who had high meat consumption and no/low vegetables.

\section{Interactive effects of lifestyle factors on CVD risk factors}

Table 3 indicates the adjusted ORs of CVD biomarkers by interaction of lifestyle factors. Participants who drank and smoked were more likely to have high TG (OR = 1.29; 95\% CI: $1.24-1.35 ; p \leq 0.001)$, TC (OR $=1.08 ; 95 \%$ CI: $1.04-1.12 ; p \leq 0.001)$ and CRP levels (OR $=1.24 ; 95 \%$ CI: $1.11-1.38 ; p \leq 0.001)$, and less likely to have high LDL-C $(\mathrm{OR}=0.83 ; 95 \% \mathrm{CI}: 0.79-0.86 ; p \leq 0.001)$ and blood glucose levels (OR $=0.91$; 95\% CI: 0.88-0.95; $p \leq$ $0.001)$ than those who neither drank nor smoked. Smoking and drinking negatively interacted with TC, LDL-C, HDL-C and blood glucose levels $(p \leq 0.001)$, but had an excess risk of high TG level (RERI $=0.21$; 95\% CI: $0.14-$ $0.29 ; p \leq 0.001)$ due to the interaction.

Those who drank and were engaged in physical activity were less likely to have higher $\mathrm{TC}(\mathrm{OR}=0.94 ; 95 \%$ CI: $0.90-0.97 ; p \leq 0.001)$, LDL-C (OR $=0.89 ; 95 \% \mathrm{CI}$ : $0.85-0.93 ; p \leq 0.001)$ and blood glucose levels (OR $=$ 0.95 ; $95 \%$ CI: $0.91-0.99 ; p \leq 0.05)$, but more likely to have low HDL-C level $(\mathrm{OR}=1.33$; 95\% CI: $1.26-1.41$; $p \leq 0.001)$ than the non-drinkers who were also not engaged in physical activity. Alcohol consumption and physical activity had an excess risk of high LDL-C (RERI $=0.11 ; 95 \%$ CI: $0.06-0.16 ; p \leq 0.001$ ) and high blood glucose levels (RERI $=0.05$; 95\% CI: 0.01-0.11; $p \leq$ $0.05)$ due to the interaction, but had a reduced risk of low HDL-C level (RERI $=-0.14 ; 95 \% \mathrm{CI}$ : $0.24-0.05$; $p \leq 0.01)$.

Participants who were consuming alcohol and were eating a vegetable-rich diet were less likely to have high TG $(\mathrm{OR}=0.91 ; 95 \% \mathrm{CI}: 0.86-0.95 ; p \leq 0.001)$, TC $(\mathrm{OR}=$ 0.92; 95\% CI: 0.89-0.96; $p \leq 0.001)$, LDL-C (OR = 0.75; 95\% CI: $0.72-0.79 ; p \leq 0.001)$ and blood glucose levels $(\mathrm{OR}=0.65$; 95\% CI: $0.62-0.68 ; p \leq 0.001)$, and less likely to have low HDL-C level (OR =0.70; 95\% CI: 0.66-0.75; $p \leq 0.001)$ than those who drank and had no or low intake of vegetables. Consumption of alcohol and vegetable-rich diet had an excess risk of high LDL-C (RERI $=0.08$; 95\% CI: 0.03-0.13; $p \leq 0.01$ ) and low HDL$C$ levels (RERI $=0.06$; 95\% CI: $0.001-0.13 ; p \leq 0.05$ ) due to the interaction, but a reduced risk of high TG level $($ RERI $=-0.10$; 95\% CI: $-0.17--0.04 ; p \leq 0.001)$.

Smokers who were engaged in physical activity were more likely to have high TG $(\mathrm{OR}=1.28$; 95\% CI: $1.22-$ $1.34 ; p \leq 0.001)$, LDL-C (OR $=1.05 ; 95 \% \mathrm{CI}: 1.00-1.10$; $p \leq 0.05), \mathrm{CRP}(\mathrm{OR}=1.16 ; 95 \% \mathrm{CI}: 1.03-1.31 ; p \leq 0.05)$ and blood glucose levels $(\mathrm{OR}=1.61 ; 95 \% \mathrm{CI}$ : $1.54-1.68$; $p \leq 0.001)$, and low HDL-C level $(\mathrm{OR}=2.40 ; 95 \% \mathrm{CI}$ : $1.20-1.51 ; p \leq 0.001)$ than non-smokers who did not 
Table 2 Unadjusted and adjusted odd ratios and 95\% confidence interval of cardiovascular disease risk factors by lifestyle factors

\begin{tabular}{|c|c|c|c|c|c|c|}
\hline & \multicolumn{6}{|c|}{ Odds ratio (95\% confidence interval) } \\
\hline & $\begin{array}{l}\mathrm{TG} \geq 1.695 \\
\mathrm{mmol} / \mathrm{L}\end{array}$ & $\begin{array}{l}\mathrm{TC} \geq 5.18 \\
\mathrm{mmol} / \mathrm{L}\end{array}$ & $\begin{array}{l}\mathrm{LDL}-\mathrm{C} \geq 2.59 \\
\mathrm{mmol} / \mathrm{L}\end{array}$ & $\begin{array}{l}\mathrm{HDL}-\mathrm{C} \leq 1.036 \\
\mathrm{mmol} / \mathrm{L}\end{array}$ & $\begin{array}{l}\mathrm{CRP} \geq 9.52 \\
\mathrm{nmol} / \mathrm{L}\end{array}$ & $\begin{array}{l}\mathrm{BG} \geq 5.556 \\
\mathrm{mmol} / \mathrm{L}\end{array}$ \\
\hline \multicolumn{7}{|l|}{ Unadjusted model } \\
\hline \multicolumn{7}{|l|}{ Smoking (Ref: Non-smoker) } \\
\hline Inhale secondhand smoke & $1.08(1.01,1.16)^{*}$ & $1.06(1.00,1.13)^{*}$ & $1.05(0.98,1.12)$ & $1.22(1.11,1.35)^{* * *}$ & $1.00(0.83,1.21)$ & $1.11(1.04,1.18)$ \\
\hline Quit smoking & $\begin{array}{l}1.70(1.62 \\
1.78)^{* * *}\end{array}$ & $1.06(1.01,1.11)^{*}$ & $1.26(1.20,1.33)^{* * *}$ & $1.92(1.80,2.04)^{* * *}$ & $\begin{array}{l}1.31(1.15 \\
1.49)^{* * *}\end{array}$ & $2.02(1.93,2.11)$ \\
\hline Smoker & $\begin{array}{l}2.10(2.03 \\
2.17)^{* * *}\end{array}$ & $1.03(1.01,1.07)^{*}$ & $1.18(1.14,1.22)^{* * *}$ & $2.88(2.76,3.00)^{* * *}$ & $\begin{array}{l}1.42(1.30 \\
1.55)^{* * *}\end{array}$ & $\underset{* * *}{2.48}(2.41,2.56)$ \\
\hline \multicolumn{7}{|c|}{ Drinking alcohol (Ref: Not drinking) } \\
\hline$\leq 1$ glass/week & $\begin{array}{l}1.22(1.17 \\
1.26)^{* * *}\end{array}$ & $0.98(0.95,1.01)$ & $1.06(1.02,1.10)^{* *}$ & $1.58(1.51,1.66)^{* * *}$ & $1.09(0.98,1.21)$ & $\prod_{* * *}^{1.35}(1.30,140)$ \\
\hline 2-3 glasses/week & $\begin{array}{l}1.77(1.68 \\
1.86)^{* * *}\end{array}$ & $\begin{array}{l}1.08(1.03 \\
1.13)^{* *}\end{array}$ & $1.02(0.97,1.08)$ & $1.60(1.50,1.71)^{* * *}$ & $\begin{array}{l}1.26(1.10 \\
1.44)^{* * *}\end{array}$ & $\begin{array}{l}1.63(1.55 \\
1.70)^{* * *}\end{array}$ \\
\hline$\geq 4$ glasses/week & $\begin{array}{l}2.39(2.26 \\
2.52)^{* * *}\end{array}$ & $\begin{array}{l}1.15(1.09 \\
1.21)^{* * *}\end{array}$ & $1.04(0.98,1.10)$ & $1.77(1.65,1.90)^{* * *}$ & $\begin{array}{l}1.46(1.27 \\
1.68)^{* * *}\end{array}$ & $\begin{array}{l}1.84(1.75 \\
1.94)^{* * *}\end{array}$ \\
\hline \multicolumn{7}{|l|}{ Physical activity (Ref: None) } \\
\hline$\leq 2 \mathrm{~h} /$ week & $\begin{array}{l}1.14(1.10 \\
1.18)^{* * *}\end{array}$ & $\begin{array}{l}0.91(0.89 \\
0.94)^{* * *}\end{array}$ & $1.02(0.98,1.05)$ & $1.86(1.78,1.94)^{* * *}$ & $1.08(0.99,1.18)$ & $\begin{array}{l}1.29(1.25 \\
1.32)^{* * *}\end{array}$ \\
\hline 3-6 h/week & $\begin{array}{l}1.06(1.02 \\
1.10)^{* *}\end{array}$ & $\begin{array}{l}0.96(0.93 \\
0.98)^{* *}\end{array}$ & $1.03(0.99,1.07)$ & $1.67(1.59,1.75)^{* * *}$ & $0.97(0.87,1.07)$ & $\begin{array}{l}1.25(1.21 \\
1.29)^{* * *}\end{array}$ \\
\hline$\geq 7 \mathrm{~h} /$ week & $0.96(0.90,1.01)$ & $0.99(0.95,1.04)$ & $1.10(1.04,1.16)^{* * *}$ & $1.47(1.37,1.58)^{* * *}$ & $0.99(0.85,1.15)$ & $\begin{array}{l}1.19(1.13 \\
1.25)^{* * *}\end{array}$ \\
\hline \multicolumn{7}{|c|}{ Diet (Ref: No/low vegetables + high meat) } \\
\hline $\begin{array}{l}\text { Moderate (vegetables + } \\
\text { meat) }\end{array}$ & $\begin{array}{l}0.87(0.84 \\
0.90)^{* * *}\end{array}$ & $\begin{array}{l}0.95(0.93 \\
0.98)^{* *}\end{array}$ & $0.91(0.88,0.94)^{* * *}$ & $0.81(0.78,0.85)^{* * *}$ & $\begin{array}{l}0.89(0.81 \\
0.97)^{* *}\end{array}$ & $\begin{array}{l}0.83(0.81 \\
0.86)^{* * *}\end{array}$ \\
\hline $\begin{array}{l}\text { High vegetables }+ \text { no/low } \\
\text { meat }\end{array}$ & $\begin{array}{l}0.73(0.71 \\
0.76)^{* * *}\end{array}$ & $\begin{array}{l}0.88(0.85 \\
0.90)^{* * *}\end{array}$ & $0.80(0.78,0.83)^{* * *}$ & $0.67(0.64,0.70)^{* * *}$ & $\begin{array}{l}0.88(0.81 \\
0.96)^{* *}\end{array}$ & $\begin{array}{l}0.67(0.65 \\
0.70)^{* * *}\end{array}$ \\
\hline \multicolumn{7}{|l|}{ Adjusted model $^{a}$} \\
\hline \multicolumn{7}{|l|}{ Smoking (Ref: Non-smoker) } \\
\hline Inhale secondhand smoke & $0.97(0.90,1.05)$ & $1.05(0.99,1.12)$ & $1.04(0.97,1.12)$ & $1.12(1.01,1.24)^{*}$ & $0.97(0.80,1.18)$ & $1.05(0.98,1.12)$ \\
\hline Quit smoking & $1.06(1.01,1.12)^{*}$ & $1.04(0.99,1.10)$ & $1.02(0.96,1.08)$ & $0.98(0.91,1.04)$ & $1.11(0.97,1.28)$ & $1.03(0.98,1.08)$ \\
\hline Smoker & $\begin{array}{l}1.44(1.38 \\
1.50)^{* * *}\end{array}$ & $\begin{array}{l}1.08(1.04 \\
1.12)^{* * *}\end{array}$ & $1.03(0.99,1.08)$ & $1.60(1.52,1.68)^{* * *}$ & $\begin{array}{l}1.33(1.19 \\
1.47)^{* * *}\end{array}$ & $\begin{array}{l}1.49(1.43 \\
1.55)^{* * *}\end{array}$ \\
\hline \multicolumn{7}{|c|}{ Drinking alcohol (Ref: Not drinking) } \\
\hline$\leq 1$ glass/week & $\begin{array}{l}0.88(0.85 \\
0.92)^{* * *}\end{array}$ & $0.98(0.94,1.01)$ & $0.90(0.87,0.94)^{* * *}$ & $0.83(0.79,0.88)^{* * *}$ & $0.93(0.84,1.04)$ & $\begin{array}{l}0.78(0.75 \\
0.81)^{* * *}\end{array}$ \\
\hline 2-3 glasses/week & $1.01(0.96,1.07)$ & $1.06(1.01,1.12)^{*}$ & $0.79(0.75,0.84)^{* * *}$ & $0.63(0.59,0.68)^{* * *}$ & $1.04(0.90,1.20)$ & $\begin{array}{l}0.67(0.63 \\
0.71)^{* * *}\end{array}$ \\
\hline$\geq 4$ glasses/week & $\begin{array}{l}1.25(1.18 \\
1.33)^{* * *}\end{array}$ & $\begin{array}{l}1.14(1.08 \\
1.20)^{* * *}\end{array}$ & $0.79(0.74,0.85)^{* * *}$ & $0.64(0.59,0.69)^{* * *}$ & $\begin{array}{l}1.22(1.05 \\
1.42)^{* *}\end{array}$ & $\begin{array}{l}0.68(0.64 \\
0.72)^{* * *}\end{array}$ \\
\hline \multicolumn{7}{|l|}{ Physical activity (Ref: None) } \\
\hline$\leq 2 \mathrm{~h} /$ week & $\begin{array}{l}1.11(1.07 \\
1.15)^{* * *}\end{array}$ & $\begin{array}{l}0.92(0.89 \\
0.95)^{* * *}\end{array}$ & $1.03(0.99,1.06)$ & $1.94(1.85,2.03)^{* * *}$ & $1.10(1.01,1.21)^{*}$ & $\begin{array}{l}1.34(1.29 \\
1.38)^{* * *}\end{array}$ \\
\hline 3-6 h/week & $1.04(1.00,1.08)^{*}$ & $\begin{array}{l}0.93(0.90 \\
0.96)^{* * *}\end{array}$ & $1.00(0.96,1.04)$ & $1.73(1.65,1.82)^{* * *}$ & $0.93(0.84,1.03)$ & $\begin{array}{l}1.25(1.20 \\
1.29)^{* * *}\end{array}$ \\
\hline$\geq 7 \mathrm{~h} /$ week & $\begin{array}{l}0.86(0.81 \\
0.91)^{* * *}\end{array}$ & $\begin{array}{l}0.88(0.84 \\
0.93)^{* * *}\end{array}$ & $0.97(0.91,1.02)$ & $1.45(1.35,1.57)^{* * *}$ & $\begin{array}{l}0.79(0.68 \\
0.92)^{* *}\end{array}$ & $1.07(1.01,1.13)^{*}$ \\
\hline \multicolumn{7}{|c|}{ Diet (Ref: No/low vegetables + high meat) } \\
\hline Moderate (vegetables + & $0.97(0.94,1.01)$ & $0.95(0.92$ & $0.91(0.88,0.94)^{* * *}$ & $0.93(0.89,0.97)^{* *}$ & $0.91(0.83,0.99)^{*}$ & $0.91(0.88$ \\
\hline
\end{tabular}


Table 2 Unadjusted and adjusted odd ratios and 95\% confidence interval of cardiovascular disease risk factors by lifestyle factors (Continued)

\begin{tabular}{lllllll}
\hline & \multicolumn{2}{l}{ Odds ratio (95\% confidence interval) } & & \\
\cline { 2 - 7 } & $\begin{array}{l}\mathrm{TG} \geq 1.695 \\
\mathrm{mmol} / \mathrm{L}\end{array}$ & $\begin{array}{l}\mathrm{TC} \geq 5.18 \\
\mathrm{mmol} / \mathrm{L}\end{array}$ & $\begin{array}{l}\mathrm{LDL}-\mathrm{C} \geq 2.59 \\
\mathrm{mmol} / \mathrm{L}\end{array}$ & $\begin{array}{l}\mathrm{HDL}-\mathrm{C} \leq 1.036 \\
\mathrm{mmol} / \mathrm{L}\end{array}$ & $\begin{array}{l}\mathrm{CRP} \geq 9.52 \\
\mathrm{nmol} / \mathrm{L}\end{array}$ & $\begin{array}{l}\mathrm{BG} \geq 5.556 \\
\mathrm{mmol} / \mathrm{L}\end{array}$ \\
\hline meat) & & $0.97)^{* * *}$ & & & $0.94)^{* * *}$ \\
High vegetables + no/low & $0.94(0.91$, & $0.85(0.83$, & $0.81(0.78,0.84)^{* * *}$ & $0.92(0.88,0.97)^{* * *}$ & $0.92(0.84,1.01)$ & $0.84(0.81$, \\
meat & $0.97)^{* * *}$ & $0.88)^{* * *}$ & & & $0.87)^{* * *}$ \\
\hline
\end{tabular}

${ }^{a}$ The model was adjusted for smoking, drinking, physical activity, diet, gender, age, education, marital status, history of cardiovascular disease, waist circumference, systolic blood pressure and diastolic blood pressure

TG Triglycerides, TC Total cholesterol, LDL-C Low-density lipoprotein-cholesterol, HDL-C High-density lipoprotein-cholesterol, CRP C-reactive protein, BG Blood glucose

${ }^{*} p \leq 0.05 ;{ }^{* *} p \leq 0.01 ;{ }^{* * *} p \leq 0.001$

exercise. The interaction between smoking and physical activity had an excess risk of low HDL-C (RERI $=0.36$; 95\% CI: $0.23-0.49 ; p \leq 0.001)$ and high blood glucose levels (RERI $=0.11 ; 95 \%$ CI: $0.03-0.19 ; p \leq 0.01$ ), but had a reduced risk of high TG $(\mathrm{RERI}=-0.10 ; 95 \% \mathrm{CI}$ : -0.17 $-\quad 0.03 ; p \leq 0.01$ ) and high TC levels (RERI $=-0.06$; 95\% CI: $-0.12--0.029 ; p \leq 0.05)$. Smokers who consumed a diet high in vegetables were still more likely to have high TG (OR $=1.17$; 95\% CI: $1.11-1.23 ; p \leq 0.001)$, blood glucose (OR $=1.09$; 95\% CI: $1.05-1.15 ; p \leq 0.001)$, and low HDL-C levels $(\mathrm{OR}=1.24 ; 95 \% \mathrm{CI}$ : $1.17-1.32$; $p \leq 0.001)$, but less likely to have high $\mathrm{TC}(\mathrm{OR}=0.95$; 95\% CI: $0.91-0.99 ; p \leq 0.05)$ and LDL-C levels $(\mathrm{OR}=$ $0.91 ; 95 \%$ CI: $0.87-0.95 ; p \leq 0.001)$ than non-smokers who consumed a diet with no or low vegetables. Smoking and vegetable-rich diet had a reduced risk of high TG (RERI $=-0.11 ; 95 \%$ CI: $-0.18--0.04 ; p \leq 0.01$ ), high blood glucose levels (RERI $=-0.14 ; 95 \%$ CI: -0.21 $--0.07 ; p \leq 0.001)$ and low HDL-C (RERI $=-0.10$; 95\% CI: $-0.19--0.01 ; p \leq 0.05)$ in an additive scale.

\section{Discussion}

Our study explored the associations between lifestyle factors and CVD risk factors, and further assessed the interactive effects of lifestyle factors on CVD risk factors. Consistent with the findings of the previous studies [1517, 31-33], our study found that smokers were more likely to have high levels of TG, TC, CRP and blood glucose and low HDL-C level, which raises the risk of CVD in smokers. The mechanism of abnormal lipid profiles in smokers may be due to an increased release of catecholamine which causes elevated free fatty acids and leads to a decrease in HDL-C level and a surge in LDL-C level [34]. In the present study, we also found that participants who drank alcohol had elevated levels of TG, TC and CRP, but were less likely to have high LDL-C, blood glucose and low HDL-C levels. The results on TG, LDL$\mathrm{C}$, blood glucose and HDL-C levels in drinkers are similar to other studies $[35,36]$. On the other hand, CRP level in drinkers was dependent on the amount of alcohol consumed as shown in the previous study that CRP level was elevated among heavy drinkers [37]. However, an animal study observed the contrary results regarding the effect of alcohol consumption on blood TG and TC levels [38].

Our results revealed those who had physical activity were more likely to have low TG, TC and CRP levels, which were consistent with the previous studies [12, 39], but were more likely to have low HDL-C level and high blood glucose level. The finding on negative effect of exercise on HDL-C level may be because of exercise intensity and volume, gender disparity and population difference. Exercise intensity and volume required to elevate $\mathrm{HDL}-\mathrm{C}$ level were more for women than those for men because HDL-C level is usually higher in women than that in men [40]. Higher HDL-C level was found in physically active women compared with that in sedentary women, but not in men [13]. High blood glucose level was found in those who had physical activity, and the result was possibly because of the variations in exercise timing, duration, intensity and type which were not recorded in our study [12, 14]. Additionally, our results supported the findings of other studies that participants with a high vegetable diet were less likely to have high TG, TC, LDL-C and blood glucose levels, and low HDL-C level than those who consumed a high meat diet [41-43].

Our study found that smoking and alcohol consumption had a negative interaction on TC, LDL-C, blood glucose and HDL-C levels. The reduced risk effect by the interaction of smoking and alcohol consumption was predominantly contributed from alcohol per se. Our findings observed those who consumed alcohol without smoking were less likely to have high blood glucose level and low HDL-C level, but smokers who did not consume alcohol were more likely to have high concentrations of all CVD biomarkers and low HDL-C level. However, smoking and alcohol consumption had a positive interaction on TG level, which was possibly attributed to smoking. Our study showed that smokers who did not consume alcohol were more likely to have raised TG level, which was contrary to the result that alcohol 


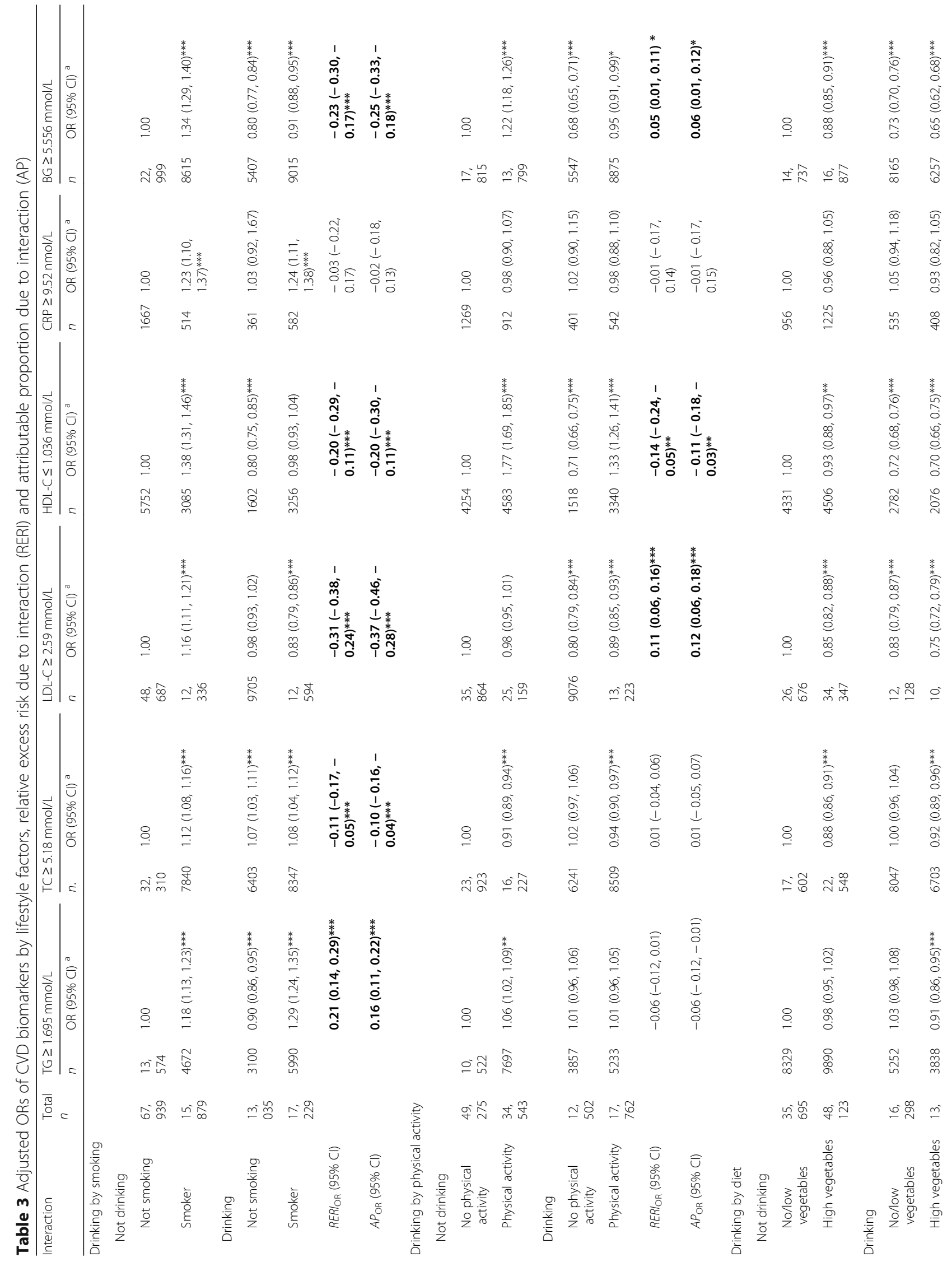




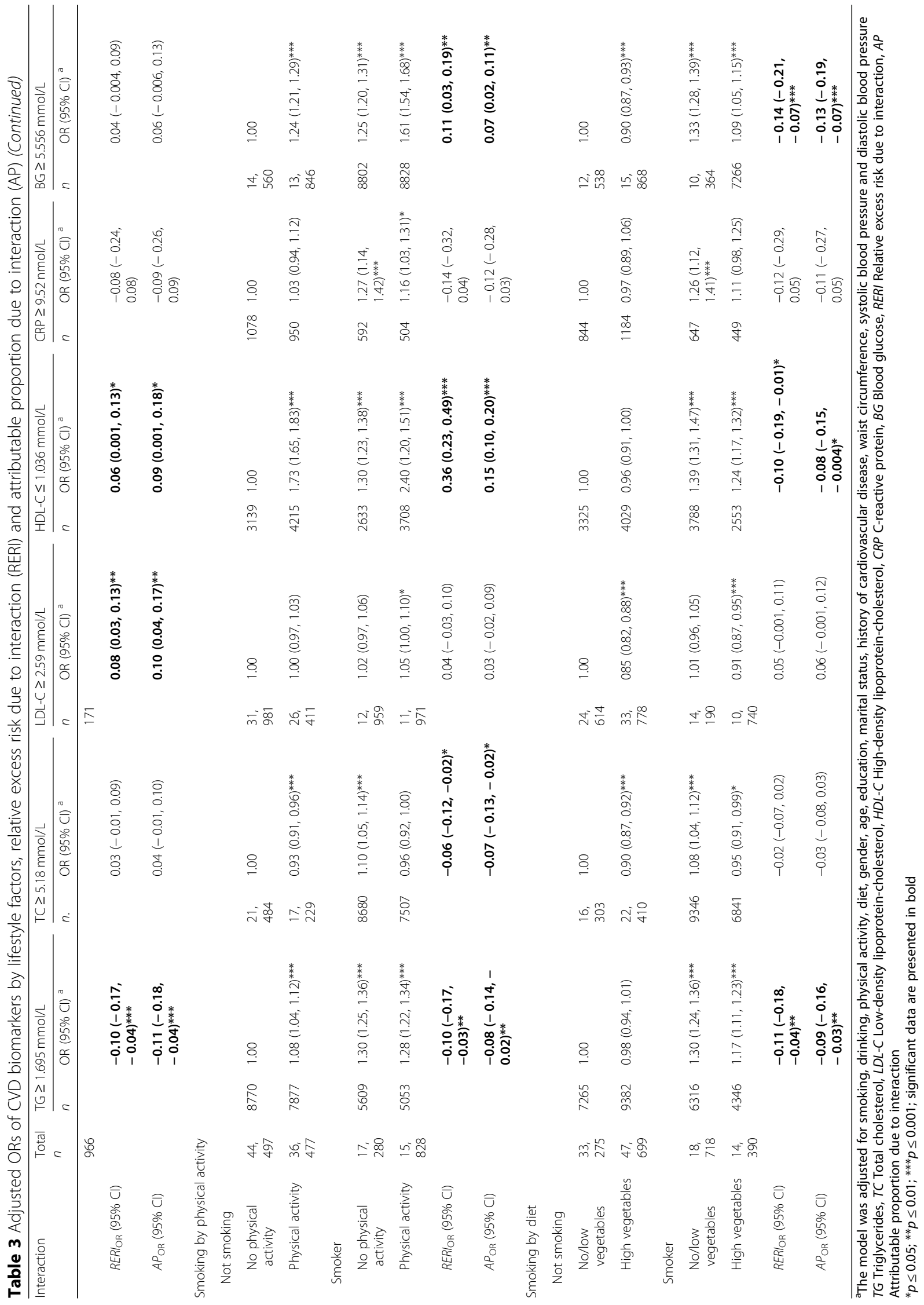


consumers who did not smoke were less likely to have high TG level.

Those who consumed alcohol and were engaged in physical activity had an excess risk on LDL-C and blood glucose levels due to the interaction. The reason for the positive interaction between alcohol consumption and physical activity was possibly because of exercise intensity and volume as well as gender disparity discussed earlier [12-14, 40]. However, alcohol consumption and physical activity reduced the risk of low HDL-C level mainly because physically active participants who did not consume alcohol were less likely to have low HDL-C level.

The consumption of alcohol and vegetable-rich diet revealed an extra risk on LDL-C and HDL-C levels, but alcohol or vegetable consumers was less likely to have high LDL-C and low HDL-C levels. The interaction between alcohol and vegetable-rich diet is still not clear. A significant additive effect of alcohol on postprandial TG level was observed as it accompanied a meal high in saturated fat [44]. On the other hand, alcohol had cholesterol-lowering effect as a result of the action of alcohol on the gastrointestinal tract [45]. More studies are still required to explain the interaction between alcohol and vegetable consumption.

We found a positive interaction between smoking and physical activity on HDL-C and blood glucose levels, but a reduced risk on TG and TC levels. Those who smoked but had physical activity were 2.4 times more likely to have low HDL-C level, and 1.6 times more likely to have high blood glucose level. Our results indicated that smokers who had physical activity were 1.3 times more likely to have high TG level. However, the interaction was negative on TG level between smoking and physical activity which needs further studies to explain this finding, though physically active participants who did not smoke had lower TC level. Additionally, smokers who consumed a vegetable-rich diet reduced the risk of high TG and blood glucose levels as well as low HDL-C level possibly due to the consumption of vegetables, which was consistent with our finding that those who consumed a diet high in vegetables and did not smoke were less likely to have high TG and blood glucose levels and low HDL-C level.

\section{Strengths and limitations}

This is the first study, to the best of our knowledge, to explore the interactive effect of lifestyle factors on CVD risk factor in middle-aged adults and elderly. The sample size collected for 10 years was large, and we may be able to generalize the findings to the population of middleaged adults and elderly in Taiwan. The variables included not only lifestyle factors but several biochemical measurements. However, the cross-sectional data reduced the possibility of causal assertions, and a cohort study or randomized controlled trial design would be appropriate for causal association. Second, our assessment of lifestyle factors may not have been comprehensive enough to adjust for associated confounding factors such as the type and intensity of exercise. Finally, data were collected by one institution that has branches in major cities of Taiwan, but people with different demographic characteristics might not have visited the institution. Therefore, one needs caution in interpreting our results.

\section{Conclusions}

Smoking and drinking are associated with higher blood lipids and CRP. Whereas physical activity is correlated with lower blood lipids and CRP, and vegetable-rich diet is correlated with lower blood lipids and glucose. Smoking, drinking, physical activity and vegetable-rich diet have an additive interaction on blood lipids and glucose. In light of these findings, public health promotion should therefore consider multifaceted promotional activities that are likely to make a positive impact on the health status of the Taiwanese population. Further research is also needed that will include other undetermined lifestyle factors and CVD risk factors, and to understand the mechanisms underlying these interactions of lifestyle factors before developing efficient and effective primary prevention strategies for CVD.

\section{Abbreviations \\ AP: Attributable proportion due to interaction; BG: Blood glucose; BMI: Body mass index; CRP: C-reactive protein; CVD: Cardiovascular disease; HDL- \\ C: High-density lipoprotein-cholesterol; LDL-C: Low-density lipoprotein- cholesterol; RERI: Relative excess risk due to interaction; TC: Total cholesterol; TG: Triglycerides}

\section{Acknowledgements}

Not applicable.

\section{Authors' contributions}

MAM and POO designed research; MAM, POO and JCJC conducted the research; $\mathrm{CYH}$ managed dataset and retrieved data; POO, MAM, JCJC and $\mathrm{CYH}$ were responsible for data analysis and interpretation; MAM and POO wrote the paper draft; All authors had primary responsibility for final content, read and approved the final manuscript.

\section{Funding}

None of the authors received funding for this study.

\section{Availability of data and materials}

The data are available from Mei Jau (MJ) Health Management Institute, but restrictedly apply to the availability of these data, and are not publicly available. Data are only available from the authors upon reasonable request and with permission of MJ Health Management Institute.

\section{Ethics approval and consent to participate}

This study was approved by the Taipei Medical University-Joint Institutional Review Board (TMU-JIRB no. 201503016). An informed consent was signed by the participants before health screening to agree that anonymized data would be used only for academic purpose.

Consent for publication

Not applicable. 


\section{Competing interests}

The authors declare that they have no competing interests.

\section{Author details}

'Department of Human Nutrition and Dietetics, School of Medicine and Health Sciences, Kabarak University, Kabarak, Nakuru, Kenya. ${ }^{2}$ Department of Public Health, School of Health Sciences, University of Eastern Africa, Baraton, Eldoret, Kenya. ${ }^{3}$ Master Programs in Public Health and Global Health, School of Health Sciences, University of Eastern Africa, Baraton, Eldoret, Kenya. ${ }^{4}$ Department of Information Management, National Taipei University of Nursing and Health Sciences, Taipei, Taiwan. ${ }^{5}$ Master Program in Global Health and Development, College of Public Health, Taipei Medical University, Taipei, Taiwan. ${ }^{6}$ School of Nutrition and Health Sciences, College of Nutrition, Taipei Medical University, 250 Wu-Hsing Street, Taipei 110, Taiwan. ${ }^{7}$ Nutrition Research Center, Taipei Medical University Hospital, Taipei, Taiwan.

\section{Received: 12 June 2019 Accepted: 25 September 2019} Published online: 22 October 2019

\section{References}

1. Hsiao AJ, Chen LH, Lu TH. Ten leading causes of death in Taiwan: a comparison of two grouping lists. J Formos Med Assoc. 2015;114(8):679-80.

2. Health Promotion Administration, Ministry of Health and Welfare. 2016 annual report of health promotion administration. Taipei City: Health Promotion Administration, Ministry of Health and Welfare; 2016.

3. Yusuf S, Hawken S, Ounpuu S, Dans T, Avezum A, Lanas F, et al. Effect of potentially modifiable risk factors associated with myocardial infarction in 52 countries (the INTERHEART study): case-control study. Lancet. 2004; 364(9438):937-52.

4. Farhud DD. Impact of lifestyle on health. Iran J Public Health. 2015;44(11): $1442-4$

5. Torpy JM, Burke AE, Glass RM. Coronary heart disease risk factors. JAMA. 2009;302(21):2388. https://doi.org/10.1001/jama.302.21.2388.

6. Grundy SM, Bazzarre T, Cleeman J, D'Agostino RB Sr, Hill M, Houston-Miller $\mathrm{N}$, et al. Prevention conference $\mathrm{V}$ : beyond secondary prevention: identifying the high-risk patient for primary prevention: medical office assessment: writing group I. Circulation. 2000;101(1):E3-E11.

7. Li YH, Ueng KC, Jeng JS, Charng MJ, Lin TH, Chien KL, et al. 2017 Taiwan lipid guidelines for high risk patients. J Formos Med Assoc. 2017;116(4):217-48.

8. Lagrand WK, Visser CA, Hermens WT, Niessen HW, Verheugt FW, Wolbink GJ, et al. C-reactive protein as a cardiovascular risk factor: more than an epiphenomenon? Circulation. 1999;100(1):96-102.

9. Dudina A, Cooney MT, Bacquer DD, Backer GD, Ducimetière $P$, Jousilahti $P$, et al. Relationships between body mass index, cardiovascular mortality, and risk factors: a report from the SCORE investigators. Eur J Cardiovasc Prev Rehabil. 2011;18(5):731-42.

10. Haskell WL. Exercise-induced changes in plasma lipids and lipoproteins. Prev Med. 1984;13(1):23-36.

11. Huang J, Frohlich J, Ignaszewski AP. The impact of dietary changes and dietary supplements on lipid profile. Can J Cardiol. 2011;27(4):488-505.

12. Mann S, Beedie C, Jimenez A. Differential effects of aerobic exercise, resistance training and combined exercise modalities on cholesterol and the lipid profile: review, synthesis and recommendations. Sports Med. 2014; 44(2):211-21.

13. Skoumas J, Pitsavos C, Panagiotakos DB, Chrysohoou C, Zeimbekis A, Papaioannou l, et al. Physical activity, high density lipoprotein cholesterol and other lipids levels, in men and women from the ATTICA study. Lipids Health Dis. 2003:2:3

14. Colberg SR, Hernandez MJ, Shahzad F. Blood glucose responses to type, intensity, duration, and timing of exercise. Diabetes Care. 2013;36(10):e177. https://doi.org/10.2337/dc13-0965.

15. He BM, Zhao SP, Peng ZY. Effects of cigarette smoking on HDL quantity and function: implications for atherosclerosis. J Cell Biochem. 2013;114(11):2431-6.

16. Komiyama M, Wada H, Ura S, Yamakage H, Satoh-Asahara N, Shimatsu A, et al. Analysis of factors that determine weight gain during smoking cessation therapy. PLoS One. 2013;8(8):e72010. https://doi.org/10.1371/ journal.pone.0072010.

17. Gossett LK, Johnson HM, Piper ME, Fiore MC, Baker TB, Stein JH. Smoking intensity and lipoprotein abnormalities in active smokers. J Clin Lipidol. 2009;3(6):372-8.
18. Friedewald WT, Levy RI, Fredrickson DS. Estimation of the concentration of low-density lipoprotein cholesterol in plasma, without use of the preparative ultracentrifuge. Clin Chem. 1972;18(6):499-502.

19. McPherson RA, Pincus MR. Henry's clinical diagnosis and management by laboratory methods. Philadelphia: Elsevier Health Sciences; 2011.

20. Pearson TA, Mensah GA, Alexander RW, Anderson JL, Cannon RO 3rd, Criqui $M$, et al. Markers of inflammation and cardiovascular disease: application to clinical and public health practice: a statement for healthcare professionals from the Centers for Disease Control and Prevention and the American Heart Association. Circulation. 2003;107(3):499-511.

21. MJ Group. MJ Health Screening Center Questionnaire QR-121-1 MJ2011.061104TW. http://www.mjlife.com/index.aspx?lang=eng\&fn=mj. Acessed 6 Feb 2016.

22. Whelton PK, Carey RM, Aronow WS, Casey DE Jr, Collins KJ, Dennison Himmelfarb C, et al. 2017 ACC/AHA/AAPA/ABC/ACPM/AGS/APhA/ASH/ ASPC/NMA/PCNA guideline for the prevention, detection, evaluation, and management of high blood pressure in adults: executive summary: a report of the American College of Cardiology/American Heart Association task force on clinical practice guidelines. Circulation. 2018;138(17):e426-83.

23. Department of Health. Identification, evaluation, and treatment of overweight and obesity in adults in Taiwan. Washington, DC: Department of Health; 2003.

24. Richardson DB, Kaufman JS. Estimation of the relative excess risk due to interaction and associated confidence bounds. Am J Epidemiol. 2009;169(6): 756-60.

25. Kalilani L, Atashili J. Measuring additive interaction using odds ratios. Epidemiol Perspect Innov. 2006;3:5.

26. VanderWeele TJ. Reconsidering the denominator of the attributable proportion for interaction. Eur J Epidemiol. 2013;28(10):779-84.

27. VanderWeele TJ. Causal interactions in the proportional hazards model. Epidemiology. 2011;22(5):713-7.

28. Hosmer DW, Lemeshow S. Confidence interval estimation of interaction. Epidemiology. 1992;3(5):452-6.

29. Zou GY. On the estimation of additive interaction by use of the four-by-two table and beyond. Am J Epidemiol. 2008;168(2):212-24.

30. StataCorp. Stata statistical software: release 13. College Station: StataCorp LP; 2013.

31. Khurana M, Sharma D, Khandelwal PD. Lipid profile in smokers and tobacco chewers-a comparative study. J Assoc Physicians India. 2000;48(9):895-7.

32. Ohsawa M, Okayama A, Nakamura M, Onoda T, Kato K, Itai K, et al. CRP levels are elevated in smokers but unrelated to the number of cigarettes and are decreased by long-term smoking cessation in male smokers. Prev Med. 2005;41(2):651-6.

33. Frati $A C$, Iniestra F, Ariza CR. Acute effect of cigarette smoking on glucose tolerance and other cardiovascular risk factors. Diabetes Care. 1996;19(2):112-8.

34. Chelland Campbell S, Moffatt RJ, Stamford BA. Smoking and smoking cessation-the relationship between cardiovascular disease and lipoprotein metabolism: a review. Atherosclerosis. 2008;201(2):225-35.

35. Bessembinders $K$, Wielders J, van de Wiel A. Severe hypertriglyceridemia influenced by alcohol (SHIBA). Alcohol Alcohol. 2011;46(2):113-6.

36. Volcik KA, Ballantyne CM, Fuchs FD, Sharrett AR, Boerwinkle E. Relationship of alcohol consumption and type of alcoholic beverage consumed with plasma lipid levels: differences between whites and African Americans of the ARIC study. Ann Epidemiol. 2008;18(2):101-7.

37. Imhof A, Woodward M, Doering A, Helbecque N, Loewel H, Amouyel P, et al. Overall alcohol intake, beer, wine, and systemic markers of inflammation in western Europe: results from three MONICA samples (Augsburg, Glasgow, Lille). Eur Heart J. 2004;25(23):2092-100.

38. Egbung GE, Atangwho IJ, Odey OD, Ndiodimma VN. The lipid lowering and cardioprotective effects of Vernonia calvoana ethanol extract in acetaminophen-treated rats. Medicines (Basel). 2017;4(4):90. https://doi.org/ 10.3390/medicines4040090.

39. Ford ES. Does exercise reduce inflammation? Physical activity and C-reactive protein among U.S. adults. Epidemiology. 2002;13(5):561-8.

40. Kokkinos PF, Fernhall B. Physical activity and high density lipoprotein cholesterol levels: what is the relationship? Sports Med. 1999;28(5):307-14.

41. Muga MA, Owili PO, Hsu CY, Rau HH, Chao JC. Association between dietary patterns and cardiovascular risk factors among middle-aged and elderly adults in Taiwan: a population-based study from 2003 to 2012. PLoS One. 2016;11(7):e0157745. https://doi.org/10.1371/journal.pone.0157745.

42. Lim JH, Lee YS, Chang HC, Moon MK, Song Y. Association between dietary patterns and blood lipid profiles in Korean adults with type 2 diabetes. $J$ Korean Med Sci. 2011;26(9):1201-8. 
43. Zhang J, Wang Z, Wang H, Du W, Su C, Zhang J, et al. Association between dietary patterns and blood lipid profiles among Chinese women. Public Health Nutr. 2016;19(18):3361-8.

44. Gross GA. Drug and alcohol abuse and cholesterol levels. J Am Osteopath Assoc. 1994;94(1):55-6 61-2.

45. Van de Wiel A. The effect of alcohol on postprandial and fasting triglycerides. Int J Vasc Med. 2012;2012:862504. https://doi.org/10.1155/ 2012/862504.

\section{Publisher's Note}

Springer Nature remains neutral with regard to jurisdictional claims in published maps and institutional affiliations.

Ready to submit your research? Choose BMC and benefit from:

- fast, convenient online submission

- thorough peer review by experienced researchers in your field

- rapid publication on acceptance

- support for research data, including large and complex data types

- gold Open Access which fosters wider collaboration and increased citations

- maximum visibility for your research: over $100 \mathrm{M}$ website views per year

At BMC, research is always in progress.

Learn more biomedcentral.com/submissions 